\title{
Inter-Induce computation and its Philosophical Foundation
}

\author{
Yasuhiro Suzuki \\ Graduate School of Informatics, Nagoya University, Furocho Chikusa \\ Nagoya City, Aichi Prefecture 464/0814, Japan* \\ E-mail:_ysuzuki@nagoya-u.jp.com \\ www.ysuzuki.info
}

\begin{abstract}
Set theory is based on the distinguishability of elements. How to recognize and identify the world is the essence of set theory. If each element cannot be identified, all the elements are one set. So the set does not make sense. The Heart Sutra is highly rational and can be interpreted mathematically. The mathematical interpretation of the Heart Sutra shows the divergence of how to discriminate. Based on this world view of Heart Sutra, we propose Inter-Induce computation, IIC as a novel calculation paradigm that does not depend on set theory. This paper gives an overview and philosophical foundation of IIC.
\end{abstract}

Keywords: Inter-Induced computation, Natural computing, Foundation of Computer Science, Heart sutra

\section{Introduction}

When I started to consider natural computing, when I was studying "the origin of life" using abstract chemistry, the 5th Workshop on Life and Physicality, SOMA 5th Theme inter-relationship between "phenomenon" and "things", February 22-23, 2002, I met a priest, Shinsho Kajita, at (at Honen-in temple, Kyoto).

And he suggested to me that "life is unrelenting." I felt something related to the "calligraphy of circle" in every part of the life system and the "circle" of the Zen priest, but at that time, I could not understand the suggestion.
Through my research on the origin of life, I finally understood Kajita's "What is life?"

There are several types of the caligraphy of circles (Enso 円相 in Japanese), for example, the calligraphy of the circle of Ikkyu Zen Master is a perfect circle (as shown to the left of the Fig.1). Systematically, this "circle" is in a steady state. Also, since it is a perfect circle, I do not know where it started. That is, there is no time. On the other hand, many calligraphies of circles have "beginning" and "end" from the ink marks, and there is a flow of time (Fig.1 right) 
Even if it is a perfect circle, time will flow from one piece to the other if you cut it somewhere. "Dissipation of information" is equivalent to "breaking" a perfect circle by "breaking" a structure that is "steady-state" and creating time.

What if a highly non-linear system with unsteady
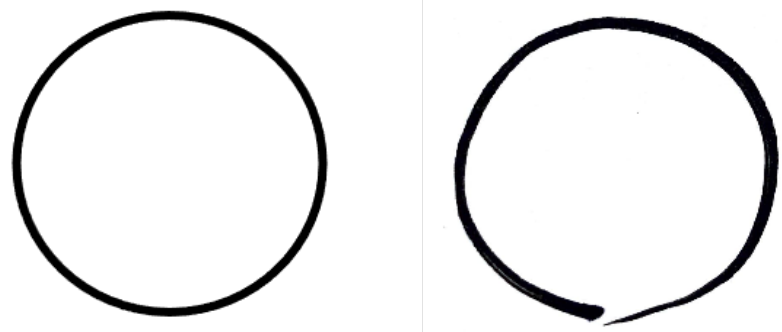

Fig. 1. Example of calligraphy of circles, left) Ikkyu Zen Master (Edo period) created a complete circle calligraphy, right) many calligraphy of circles are not complete circle, which has a "start point" as breaking point; and also density of a stroke illustrates the start-end point

(unsteady) rows (all) does not take a circular structure? If not, all systems have a circular structure in infinitedimensional state space, it isn't easy to know what the state space looks like unless you can see the entire state space. But if the system has a circular structure, and if it is low-dimensional (two-dimensional or threedimensional), we can recognize the system.

The calligraphy of a circle that is not closed is topologically a straight line. Then, the straight line disappears at the break of the calligraphy of a circle and regenerates forever. We can recognize the calligraphy of a circle because it is "circular". If it breaks out of the "hanging scroll" as a myriad of straight lines, it is difficult for us to recognize it. The calligraphy of a circle is an example of a case where a system of invariant behaviors can be recognized, such as an attractor of a chaotic dynamical system.

\section{Philosophy of Hear Sutra}

The Heart sutra (般若心経) ${ }^{1}$ is a well-known "sutra". However, its composition is rational and precise. And it is suggested that element reductionism causes bankruptcy. The latter half of the Heart Sutra is a philosophical suggestion. Still, the first half shows that the classification method diverges and becomes meaningless when the theory of element reduction is pursued.

The concept of "colour" is used in the following, but in Buddhism, "colour" corresponds to vision among the five senses. In Chinese, "not unmatch" means "same", but here "not unmatched" is regarded as inclusion. Because, if you want to show "colour, 色 and Kuh, 空 are the same", you can set "colour" = "Kuh". However, in this case, "Kuh" is not specifically shown and becomes undefined.

However, it is possible to define "Kuh" different from "colour" by regarding "Color" $\subseteq$ "Kuh" \$ and "Kuh" $\subseteq$ "Color", "Kuh" is truly included in "Color", and "Color" is truly included in "Kuh". From this equivalent relation, it is mathematically shown that "color" = "Kuh".

"Colorless Kuh, Kuhless colour" are paired. Still, regardless of their religious and philological meanings, mathematically, no matter which one is missing, the identity cannot be proved, so it is inevitable. This is the definition.

"Colorless Kuh" is the definition of the existence of "colour" and "Kuh". Although the existence of "colour" and "Kuh" is defined, this definition claims that "colour" and "Kuh" are the same, and time and dynamics do not occur from this definition. In terms of Enso, it shows the existence of Ikkyu's "completely closed perfect circle." It is the definition of the specific relationship between the two;

Color, that is (即是) Kuh, Kuh, that is (即是) color.

In Chinese, "即是, immediate correction" means "that is". Therefore, "immediate correction" can be regarded as implies, $\Rightarrow$. $\mathrm{A} \Rightarrow \mathrm{B}$ by this symbol means "A, that is, if $\mathrm{B}$ ". Mathematically, $\mathrm{A} \Rightarrow \mathrm{B}$ and $\mathrm{B} \Rightarrow \mathrm{A}$ prove that $\mathrm{A}$ and $\mathrm{B}$ are equivalent. Again, they are opposites, but they must be mathematically paired because they cannot be shown to be equivalent if either one is missing (this $\Rightarrow$ can be regarded as a map, but in category theory. May be regarded as morphism).

In the above, "colour (vision)" of the five senses is discussed, but other senses are omitted because the same discussion is repeated. From this, all "functions of the mind" are equal to "Kuh".

The construction of this concept of "Kuh" is logically clever (aside from religious discussions), and it does not define what "Kuh" is, and what is called "the function of 
the mind" is individual. It is shown that it cannot be divided into.

If all the "functions of the mind" are equal to "Kuh", the concepts that conflict with the "functions of the mind" such as life-destruction, dirt-purification, and increasedecrease are life $=$ destruction $=$ dirt $=$ purification $=$ increase = decrease. $=$ "Kuh", so everything is the same. In other words, "it cannot be distinguished".

For example, the increase/decrease of "Your juice is more than mine!" Does not hold if you accept the existence of "Kuh". Conversely, if we accept the existence of "Kuh", time-related confrontations such as life-destruction, dirt-purification, and increase-decrease do not make sense (immortality and immortality). Unclean and unclean). Also, as discussed in "colour" and "Kuh", all five senses are the same as "Kuh", so the five senses are all the same from visual $=$ auditory $=$ olfactory $=$ taste $=$ tactile $=$ "Kuh" and cannot be distinguished.

The dynamics are "unsteady", that is, unsteady, but in reality, they are all the same "Kuh", and our perception ("the function of the mind") is "Kuh" and the visual image ("colour") is projected. For example, as Jakob Johann Baron von $\mathrm{Uexku}^{2}$ ) suggests, recognition differs depending on the species even in the same field, but in reality, they are the same field.

Attractor-like mechanical structures can sometimes be seen in (low-dimensional) chaos, but their trajectories are all "solutions" of the same equation. A function does not always have a unique solution if it does not meet the Lipschitz condition. In that case, the solution can be innumerable, and the behaviors of various dynamical systems can be seen depending on the parameters. However, although they are all "unsteady" (unsteady) in terms of dynamics, they are really just "solutions" to the same equation.

On the other hand, the Heart Sutra suggests that recognizing the unchanging natural system leads to the "Kuh." This suggestion is accurate and analytical, and element-reducing science continues to make "lists" of how natural systems work, but this lengthy list, as Yuxcur suggests, can be perceived differently.

Even if a "perfect list" is obtained in the future, it is unlikely that we can understand the natural system that is unrelenting by combining it. An example with a "perfect list" is a cellular automaton. In cellular automata, components and all interactions are perfectly grasped as a finite number of state transition rules. However, that dynamics go beyond our perception and creates a new universe-like world. The universe of cellular automata is vast, and we can only observe it.

\section{Framework of Inter-Induced Computation}

Based on the Heart Sutra concept, the components of the natural system are subdivided as much as possible. In contrast to searching for the ultimate components, in the following, the components of the natural system are not subdivided but treated as they are. We propose a calculation system.

In formulating the actual nature into a computational system, Heart sutra suggested it is complicated to understand the natural system in element reductionism. It is premised that classification-analysis is impossible. Must be. In that case, it does not become a meaningful set theory. This is because all the elements are the same (one representative source). Therefore, it is necessary to change the basis for constructing a computational system fundamentally.

On the other hand, we do not fully understand the natural system, but we interact and control it to some extent. For example, when communicating with a person, it is impossible to subdivide the other person into elements and fully understand them before communicating.

However, we communicate and compose a society without completely understanding the other person. Then, as I go out with the other person, I gradually feel that I can understand the other person. However, neither knows whether the belief is correct or incorrect (because they cannot know each other's internal state accurately).

Therefore, a framework that guides the target system in the desired direction while interacting with the target, such as communication with humans, is called "inter induce computing".

In the following, the guiding side is referred to as the "inducing subject", and the guided side is referred to as the "inducing target". For the sake of simplicity, in the following, the guiding subject and the guiding target are independent, autonomous systems. It is possible to argue even if they are subordinate to each other. Still, in that 
case, specifically dependent interactions and independent interactions Since it is necessary to divide into actions and there are innumerable cases, only independent cases are dealt with.

And here is the most important point, "It is impossible to know the internal state of the other party for both mutual subjects and mutual objects by any method." The guiding subject has a "direction/state of being guided" as a solution set. Interactions that bring about change are called "effective actions", and interactions that do not change are called "ineffective actions". In the following, unless otherwise specified, effective action is called an "action".

The inducer "acts" on the induction target. The induction target changes depending on the "action" and leads to a steady-state (including a dynamic steady-state) and a normal set. The guiding subject observes this normal typeset, and if it matches the guided direction and state, it is a "correct answer", otherwise it is a "wrong answer". And from this result, a new "action" may be performed. The inducer decides whether or not to perform a new "action".

\section{Remarks}

Why is it mutual induction rather than induction?

It is impossible to know the internal state of the other party's system with which any natural / life system interacts, by any method. Therefore, for convenience, we have named the guiding subject and the guiding target. However, the "name" has no essential meaning. Therefore, the mutual induction system is essentially composed of two or more systems.

It is impossible to determine whether any natural or biological system is "inducing or being guided". For example, if I think that someone is "inducing", whether the object to be guided is "induced by me" or "in reverse, I am induced", the "action" is established in the first place. I don't know.

It is impossible to "really know (know the other party's internal state from a transcendental standpoint)".

For example, a virus needs a "host" because it cannot propagate on its own. Emerging infectious diseases such as coronavirus mutate while interacting with the "host". When the "host" is human, humans make vaccines and new drugs and "respond" to the "action" of the virus. In this case, both are the guiding subjects and the guiding targets.
If there is no object of interaction, the concept of induction/delusion does not occur. Self-reflection and delusions are also multiple systems consisting of "selfreflection / delusional subject" and "self-reflection / delusional system" created by that subject. It is a mutual induction. It is not known in principle (unless it is a transcendental existence) which system is mutual subject or mutual object, and even how many of them are unknown.

The essence of induction is that there is an interaction partner. It doesn't matter if the "other party" actually exists. To clarify this, it is called mutual induction.

\section{Isn't mutual induction a feedback control?}

The same argument applies to any control system, but when controlling, the control subject and the control target are generally clearly defined, and the internal state of the control target can be known as a state quantity such as temperature or velocity, for example., In autopoiesis, it is not possible to clearly separate the control subject and the object, but it is part of the definition of autopoiesis, and the discussion, in that case, is similar to mutual guidance. Therefore, in control, the actionresponse of the controlled object is formulated. It can be handled mathematically.

On the other hand, in mutual induction, it is premised that the guiding subject and the guiding target cannot be separated in principle. Other systems can't know the internal state of the system. It is possible to regard a part of mutual induction as feedback control, etc.. Still, from that point alone, it is an over-generalization and an error to assume that the concept of mutual induction is the same as feedback control.

\section{References}

1. Heart Sutra

2. Jakob Johann Baron von Uexküll, Streifzüge durch die Umwelten von Tieren und Menschen: Ein Bilderbuch unsichtbarer Welten, 1934 